Abstract 72 Table 1 LIT Responsiveness against various anchors stratified by FM status in SLE

\begin{tabular}{|c|c|c|c|c|c|c|c|c|c|}
\hline & $\Delta$ Category & $\mathrm{N}$ & Mean $\Delta$ LIT & SRM & ES & $\mathbf{N}$ & Mean $\Delta$ LIT & SRM & ES \\
\hline & ALL $(n=1356)$ & & & & & No FM & & & \\
\hline \multirow[t]{8}{*}{ T2T } & Improved & & & & & & & & \\
\hline & NO to ROFT & 13.00 & -8.80 & -0.43 & -0.39 & 11.00 & -5.5 & -0.26 & -0.29 \\
\hline & NO to RONT/LDAS & 122.00 & -3.40 & -0.18 & -0.14 & 119.00 & -3.4 & -0.18 & -0.14 \\
\hline & Same & & & & & & & & \\
\hline & No to NO & 102.00 & -0.80 & -0.05 & 0.04 & 97.00 & -0.9 & -0.05 & -0.04 \\
\hline & Worsened & & & & & & & & \\
\hline & RONT/LDAS to NO & 111.00 & 4.80 & 0.25 & 0.22 & 105.00 & 4.90 & 0.25 & 0.22 \\
\hline & ROFT to NO & 10.00 & 4.40 & 0.26 & 0.23 & 9.00 & 1.40 & 0.09 & 0.07 \\
\hline \multirow[t]{3}{*}{ SLAQ } & Improved $(\downarrow \geq 5)$ & 85.00 & -3.88 & -0.21 & -0.16 & 81.00 & -3.50 & -0.19 & -0.15 \\
\hline & Same & 904.00 & -1.12 & -0.07 & -0.05 & 853.00 & -1.00 & -0.06 & -0.05 \\
\hline & Worsened $(\uparrow \geq 5)$ & 68.00 & 3.05 & 0.14 & 0.14 & 65.00 & 2.80 & 0.13 & 0.13 \\
\hline \multirow[t]{3}{*}{ EQ5D } & Improved $(\uparrow \geq 10)$ & 309.00 & -7.60 & -0.40 & -0.33 & 292.00 & -7.50 & -0.41 & -0.34 \\
\hline & Same & 427.00 & -1.60 & -0.13 & -0.07 & 403.00 & -1.47 & -0.12 & -0.06 \\
\hline & Worsened $(\downarrow \leq 10)$ & 308.00 & 6.00 & 0.32 & 0.27 & 291.00 & 6.07 & 0.32 & 0.28 \\
\hline
\end{tabular}

and were included in this analysis. 40/84 (47.6\%) patients had BILAG response at 6 months. In univariate and multivariate analysis, high IFN-Score-B expression was significantly associated with clinical response (see table 1).

Conclusions This preliminary analysis suggests that assessment of IFN activity has a role in prediction of response to rituximab. A novel IFN score (Score B) was more predictive than classic ISGs (Score A). These results add to a body of work showing that IFN-Score-B predicts clinically significant outcomes independently of overall IFN activity. Future work will analyse this biomarker in a larger cohort of patients and integrate with other putative clinical and biological predictors of response.

Funding Source(s): Medical Research Council, National Institute of Health Research

\section{LUPUS IMPACT TRACKER IS RESPONSIVE TO CHANGES IN PHYSICIAN (T2T) AND PATIENT (SLAQ, EQ5D) RELEVANT OUTCOMES IN A LARGE SPANISH LUPUS REGISTRY COHORT}

${ }^{1}$ Iñigo Rua Figueroa, ${ }^{2}$ Herve Devilliers, ${ }^{3}$ Jose Maria Pego Reigosa, ${ }^{4}$ Irene Altabás Gonzále ${ }^{5}$ Javier Narváez-García, ${ }^{6}$ Francisco Javier López-Longo, ${ }^{6} J u a n$ Ovalles-Bonilla, ${ }^{7} J a i m e$ Calvo Alen, ${ }^{8}$ Maria Galindo Izquierdo, ${ }^{9}$ Antonio Fernandez Nebro, ${ }^{10}$ Mónica Ibáñez-Barcelo, ${ }^{11}$ Carlos Montilla Morales, ${ }^{12} \mathrm{M}^{a}$ Jesús García-Villanueva, ${ }^{13}$ Paloma Vela-Casasempere,

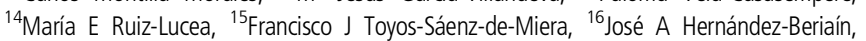
${ }^{17}$ Elvira Díez-Álvarez, ${ }^{18}$ Joel Block, ${ }^{18}$ Meenakshi Jolly. ${ }^{1}$ Hospital De Gran Canaria dr. Negrín; ${ }^{2}$ Centre Hospitalier Universitaire de Dijon; ${ }^{3}$ Complexo hospitalario Universitario Vigo; ${ }^{4}$ Hospital Do Meixoeiro; ${ }^{5}$ Hospital de Bellvitge; ${ }^{6}$ Hospital General Universitario Gregorio Marañón; ${ }^{7}$ Hospital Universitario Araba; ${ }^{8}$ Hospital Universitario 12 De Octubre; ${ }^{9}$ UGC de Reumatología, Instituto de Investigación Biomédica de Málaga (IBIMA) Hospital Regional Universitario de Málaga, Spain; ${ }^{10}$ Hospital Son Llàtzer De Palma De Mallorca; ${ }^{11}$ Hospital Clinico Universitario Salamanca; ${ }^{12}$ Hospital Ramón Y Cajal; ${ }^{13}$ Hospital General De Alicante; ${ }^{14}$ Hospital De Basurto; ${ }^{15}$ Hospital Virgen De La Macarena; ${ }^{16}$ Hospital Insular De Gran Canaria; ${ }^{17}$ Hospital De León; ${ }^{18}$ Rush University Medical Center

\subsection{6/lupus-2019-Ism.72}

Background Remission and Low Disease activity state (LDAS) are physician assessed treat to target-T2T outcomes for Systemic Lupus Erythematosus (SLE). Lupus Impact Tracker (LIT), a ten-item unidimensional patient reported tool has good psychometric properties and responds to patient reported changes in health, physician based disease activity (DA) and composite response Index (SRI). Herein we report responsiveness of LIT to changes in physician (T2T) and patient assessed outcomes (DA by SLAQ and health status (EQ5D)) among SLE patients from the largest European SLE registry- cohort.

Methods One-year longitudinal, observational, multi-center data from 1364 adult patients with SLE meeting 1997 ACR criteria were obtained from baseline and year 1 visit. This included demographics, patient reported tools (LIT, EQ5D VAS, SLAQ), SLE (activity-SLEDAI) and medications. Remission off therapy (ROFT) was defined as SLEDAI $=0$ without prednisone or Immunosuppressive/s. Remission on-therapy (RONT) was $S L E D A I=0$ and a prednisone dose $5 \mathrm{mg} /$ day and/or Immunosuppressive/s (maintenance dose). LDAS (modified) was defined as SLEDAI 4, prednisone dose $9 \mathrm{mg} /$ day and/or maintenance immunosuppressive/s. Non-optimal (NO) disease status was SLEDAI $>4$ and/or prednisone dose $>9 \mathrm{mg} /$ day and/or immunosuppressive/s in induction dose. Use of hydroxychloroquine was permitted in all groups. LIT values were compared using mixed models. Responsiveness was evaluated by standard response means (SRM) in groups with changes in DA (T2T, SLAQ) and EQ5D VAS as anchors. We did not have enough observations for stratified analysis for SLE patients with fibromyalgia.

Results 1232/1364 (90\%) were women, and 95\% were Caucasian. Mean (SD) SLEDAI and SDI were 2.6 (3.5) and 0.7 (1.1) respectively. As (i) DA was low (median 2) in LDAS, (ii) steroid use was more prevalent in RONT than LDAS, we combined RONT and LDAS into one category to analyse patient relevant differences in LIT.

LIT was responsiveness in the appropriate direction with improvement and worsening in disease activity (T2T and SLAQ) and health status (EQ5D VAS) over time. Mean LIT changes to and from NO to RONT/LDAS ranged from 3-5 (table 1), while it declined by over 8.5 with change from NO to ROFT. We had limited observations for ROFT to NO change. Mean change in LIT ranged from -3 to 3 with improvement and worsening in SLAQ, and from -7.6 to 6 with improvement and worsening in EQ5D VAS. 
Conclusions LIT responds appropriately in both directions to changes in physician (T2T) as well as patient relevant (DA and health status) outcomes among Spanish SLE patients.

Funding Source(s): None

\section{\begin{tabular}{|l|l}
\hline 73 & RHEUMATOID FACTOR IN PATIENTS WITH SYSTEMIC
\end{tabular} LUPUS ERYTHEMATOSUS}

Diana G Garate*, Demelza D Yucra, Ruth Balcazar, Hamaui Adriana, Diana Dubinsky. Sanatorio Guemes

10.1136/lupus-2019-|sm.73

Background Rheumatoid factor (RF) in SLE is found in around $25 \%$ of patients. Authors associate it with cutaneous involvement, Sicca, anti Ro $(+)$ and a protective role in glomerulonephritis (GMN), suggested by the finding of less frequent and less severe nephritis in lupus patients with $\mathrm{RF}(+)$ as in patients with RA.

The proposed protective mechanisms of RF include preventing the complement from joining immune-complexes (IC) and avoiding the IC deposit in the glomerulus.

We aimed to determine the clinical and immunological profile of SLE patients with and without RF and to determine the association between RF and GMN.

Methods Descriptive, retrospective study of SLE (SLICC 2012) patients selected from Rheumatology department of Güemes Hospital, from January 2015-August 2018.

We collected data on demographics, cutaneous-articular, renal, hematological and CNS involvement. Immunoserology: RF IgM by immunoturbidimetric $(+)>30 \mathrm{UI} / \mathrm{ml}$, anti Ro, anti La, anti Sm, anti RNP (ELISA), ANA (Hep 2), Anti DNA (Crithidia lucilliae), C3, C4, aPL at least one

Abstract 73 Table 1 Subgroup characteristics

\begin{tabular}{|l|l|l|l|}
\hline & $\begin{array}{l}\text { RF (+) } \\
\text { n 22 (\%) }\end{array}$ & $\begin{array}{l}\text { RF (-) } \\
\text { n 85 (\%) }\end{array}$ & p \\
\hline Female & $16(73)$ & $77(90)$ & 0.02 \\
Tobacco & $4(18)$ & $6(7)$ & 0.07 \\
Discoid & $0(0)$ & $9(10)^{*}$ & 0.049 \\
GMN & $6(27)$ & $28(33)$ & 0.31 \\
Ro & $18(82)^{*}$ & $35(41)$ & 0.0003 \\
La & $11(50)^{*}$ & $16(18)$ & 0.002 \\
Sm & $6(27)$ & $18(21)$ & 0.27 \\
RNP & $7(32)$ & $27(32)$ & 0.49 \\
DNA & $13(59)$ & $41(48)$ & 0.50 \\
Low complement & $17(77)$ & $66(77)$ & 0.47 \\
SICCA & $7(32)$ & $17(20)$ & 0.12 \\
aRb + & $0(0)$ & $11 / 71(15)^{*}$ & 0.04 \\
SAF & $2(9)$ & $7(8)$ & 0.43 \\
RA & $6(27)^{*}$ & $4(4)$ & 0.002 \\
S. Sjogren & $3(14)$ & $6(7)$ & 0.17 \\
\hline
\end{tabular}

\begin{tabular}{|l|l|l|l|}
\hline & \multicolumn{2}{|c|}{ RF negative } & \\
\hline & \multicolumn{1}{|c|}{ Ro + } & \multicolumn{1}{c|}{ Ro- } & \multicolumn{1}{c|}{$\boldsymbol{p}$} \\
\hline La & $13 / 35(37 \%)$ & $2 / 50(4 \%)$ & 0.00006 \\
\hline GNF & $16 / 35(46 \%)$ & $12 / 50(24 \%)$ & 0.02 \\
\hline AR & $2 / 35(6 \%)$ & $2 / 50(2 \%)$ & 0.36 \\
\hline
\end{tabular}

of the following: LI, anti 2GP1 IgG/IgM, Anti aCL IgG/ IgM.

Statistical Analysis: Epiinfo version 7.2.0.1.

Results We reviewed 147 clinical histories, 107 with RF ordered. Female 93/107 (86.9\%), mean age 41.6 years (SD \pm 13.8$)$. Disease duration 98 months $(\mathrm{SD} \pm 80.6)$. Tobacco $\begin{array}{lllllll}\text { exposure } & 10 / 107 & (9.35 \%) \text {. } & \mathrm{RF} & (+) & 22 / 107 & (20.5 \%), \\ \mathrm{RF}\end{array}$ median titer $228.8 \mathrm{IU} / \mathrm{ml}(31-2825 \mathrm{IU} / \mathrm{ml})$.

RF level in patients with GMN was $142 \mathrm{IU} / \mathrm{ml}$ (39-191), without GMN $258 \mathrm{IU} / \mathrm{ml}(31-2825) \mathrm{p}=0.46$.

RF level in patients with anti Ro (+) was $248 \mathrm{IU} / \mathrm{ml}$ (312825), Ro (-) $52.5 \mathrm{IU} / \mathrm{ml}(31-74) \mathrm{p}=0.37$.

RF (-) subgroup showed statistically significant association with female sex, discoid lesions and aPL presence. $\mathrm{RF}(+)$ was associated with $\mathrm{Ab}$ Ro, La and patients with RA. (Table 1).

Not found significant association with: rash, photosensivity, oral ulcer, joint pain, arthritis, serositis, neurologic and hematological involvement.

In those with GMN, we found a significant association with $\mathrm{Ab} \mathrm{Ro}(+)$ in the absence of RF (table 1).

Conclusions Similar RF frequency was found as the published literature. There was no association with nephritis but the mean RF titer was higher in patients without GMN.

The detection of $\mathrm{Ab}$ clusters associated with $\mathrm{RF}$ may be useful to suggest absence of renal involvement.

Funding Source(s): None

\section{RNA-SEQUENCING TO DISSECT THE ROLE OF SELECTIVE HDAC6 INHIBITION ON B CELL SIGNALING AND GERMINAL FORMATION IN LUPUS NEPHRITIS}

${ }^{1}$ Christopher M Reilly*, ${ }^{2}$ Jingjing Ren, ${ }^{3}$ Amrie Grammer, ${ }^{4}$ Peter Lipsky. ${ }^{1}$ VCOM; ${ }^{2}$ Virginia Tech; ${ }^{3}$ AMPEL Biosolutions LLC; ${ }^{4}$ AMPEL BioSolutions

10.1136/lupus-2019-Ism.74

Background Autoantibody production by plasma cells (PC) play a pivotal role in pathogenesis in of lupus nephritis (LN). The mechanism(s) of how B cells become pathogenic PC secreting autoantibody in SLE is incompletely characterized. In the current study, sought to determine if selective histone deacetylase (HDAC)6 inhibition would abrogate abnormal B cell activation in SLE.

Methods We treated 20-week-old NZB/W lupus mice with the selective HDAC6 inhibitor ACY-738 for four weeks beginning at 20 weeks-of age; at early disease. After 4 weeks-of-treatment, we used RNA-seq to determine the genetic signatures of splenocytes with and without treatment and applied signaling pathway computational analysis to reveal multiple pathways associated with $\mathrm{B}$ cell activation and differentiation in SLE that were modulated by HDAC6 inhibition.

Results Plasma cell development was abrogated as well as GC were greatly reduced. Additionally, kidney pathology was greatly reduced. When gene signature was compared to human lupus patients, the HDAC6 inhibitor treated mice showed several inflammatory pathways were decreased.

Conclusions Taken together, these studies suggest that HDAC6 inhibition decreased B cell activation signaling pathways and reduced PC differentiation in LN, suggesting that HDAC6 inhibition may represent an effective target to treat SLE.

Funding Source(s): None 